\title{
Prevalencia de trastornos de personalidad en una muestra de 115 clientes con trastornos por uso de drogas
}

\author{
Bricolo, F; Gomma, M.; Bertani, M.E.; Serpelloni, G. \\ Dipartimento delle Dipendenze. Verona. Italia. \\ Enviar correspondencia a: \\ F. Bricolo. Dipartamento delle Dipendenze. Via Germania 20. 37135 Verona (Italia). fbricolo@dronet.org
}

\section{RESUMEN}

Introducción. La presencia de trastornos psiquiátricos en pacientes con trastornos por uso de drogas (Substance Related Disorders, SRD) se conoce con el nombre de "Comorbilidad Psiquiátrica". Si por una parte existe un consenso sustancial sobre el hecho de que los trastornos de carácter, angustia y personalidad son los trastornos psiquiátricos más frecuentes en este tipo de patologías, por otra, los datos sobre la prevalencia de trastornos psiquiátricos en pacientes SRD siguen siendo contradictorios.

Objetivos. Medir la prevalencia de trastornos de personalidad (Personality Disorders, PDs) en una muestra de pacientes SRDs usando como herramienta estándar el SCID-II, un cuestionario para detectar trastornos de personalidad que consta de 117 puntos en el Eje II de DSM-IV.

Estrategia de la investigación. Se pide a 115 pacientes SRDs entre los clientes del Departamento de Adicciones, Unidad 1, de Verona, Italia, en un periodo comprendido entre 1998 y 2000, que contesten el cuestionario SCID-II.

Resultados. De los 115 clientes un 23\% eran mujeres y un $77 \%$ hombres. Tras explicarles el procedimiento correcto, más del $90 \%$ aceptaron contestar el cuestionario. En nuestra muestra de pacientes los resultados obtenidos fueron los siguientes: $52 \%$ de Paranoides, Esquizotípicos 26\%,35\% de Esquizoides, Borderline 76\%, 69\% Antisociales, 42\% Narcisistas, 10\% Histriónicos, Evitantes 35\%, Obsesivo-Compulsivos 40\%, 26\% Depresivos, Personalidad Negativa 47\%.

Límites. No pudo contarse con una población sin SRD ni/o con una población normal para poder establecer comparaciones; la participación en el protocolo y la comorbilidad de los doce PDs es más alta que en otros estudios.

Conclusión. En comparación con la mayoría de los estudios publicados, nuestra población arroja una prevalencia más elevada de PDs del grupo B, debida posiblemente a diferencias en los datos personales.

Palabras clave: comorbilidad, trastornos de personalidad, trastornos por uso de drogas.

\section{ABSTRACT}

Introduction. The presence of psychiatric disorders in Substance Related Disorders (SRD) patients is known as "Psychiatric Comorbidity". While on one side there is substantial agreement on the fact that Humor, Anxiety and Personality disorders are the psychiatric disorders which are more often found in this type of pathology, on the other side there is still discordant evidences on the prevalence of psychiatrics disorders in SRD patients.

The aim. To measure the prevalence of Personality Disorders (PDs) in a sample of SRDs patients using the golden standards instrument SCID-II, a 117 items interview for Personality Disorders in the II Axis of DSM-IV.

Search strategy. 115 SRDs patients of the clients of the Addiction Department, Unit 1 in Verona, Italy in the period from 1998 to 2000 have been asked to answer to the SCID-II interview.

Results. Out of 115 clients $23 \%$ were females and $77 \%$ males. More than $90 \%$ have accepted to answer the questionnaire after the correct procedure was explained. We have found that in our patient sample the PDs distribution is: Paranoid 52\%, Schizotipic 26\%, Schizoid 35\%, Borderline 76\%, Antisocial 69\%, Narcissistic 42\%, Histrionic 10\%, Avoidant 35\%, Obsessive-Compulsive 40\%, Depressive 26\%, Negativistic 47\%.

Limits. A control population without SRD and/or normal population is not available for comparison, the participation to the protocol and the comorbidity among the twelve PDs is higher than in other studies.

Conclusion. Our population shows a higher prevalence of PDs cluster B compared to most studies presented in literature possibly due to differences in personal data.

Key words: co morbidity, Personality Disorders, Substance Use Disorder. 
Los momentos de locura se alternaban con prolongados momentos de lucidez que me resultaban insoportables. Durante estos ataques de inconsciencia total bebía, y sólo Dios sabe en qué cantidad y con qué frecuencia. Obviamente, mis amigos achacaban la locura a la bebida y no la bebida a la locura.

\section{INTRODUCCIÓN}

$\mathbf{L}$ os primeros estudios sobre comorbilidad psiquiátrica en trastornos relacionados con el uso de drogas se realizaron en los años 80 . Rounsaville et al (1982) estudiaron una muestra de 638 adictos a los opiáceos, determinando el $69 \%$ de los diagnósticos psiquiátricos asociados. En sus estudios sobre la población dependiente de las drogas, Ross y col. (1988), señalaron un 78\% de prevalencia "lifetime". Los datos aportados por Clerici confirman esta situación: en una población de adictos a los opiáceos, el $25 \%$ no presentaba un doble diagnóstico; el $50 \%$ presentaba un diagnóstico de eje II y el $25 \%$ restante presentaba un diagnóstico añadido de eje I. Finalmente, en un estudio reciente de Brooner, realizado sobre una muestra de 716 sujetos, se ha demostrado la existencia de comorbilidad psiquiátrica en el $47 \%$ de los pacientes (para ser más exactos, en el $47 \%$ de las mujeres y en el $48 \%$ de los hombres): los diagnósticos más frecuentes fueron los comportamientos personales antisociales (en un $25 \%$ de los casos), y la depresión (en más de 15,8 \%).

Los trastornos añadidos tienen entidad gnosológica propia, pero las tipologías de los pacientes muestran diferencias relacionadas con el tipo de droga consumida, con las vías de consumo, las dosis, las situaciones sociales y relacionales y, especialmente, con los rasgos psicológicos y psiquiátricos. El $50 \%$ de los individuos presenta, de hecho, una comorbilidad entre trastornos mentales graves y abuso de drogas. Esta comorbilidad se asocia a un alto nivel de recaídas, hospitalizaciones, violencia, encarcelamientos y falta de residencia fija.

En los próximos 15 años los trabajadores clínicos, la administración y los políticos tendrán que asumir la integración de servicios de salud mental en los centros dedicados al tratamiento de drogodependencias. Será necesario que adopten métodos que permitan reforzar los tratamientos adecuados tanto para los trastornos mentales como para los relacionados con el consumo de drogas.

El diagnóstico precoz y articulado de la comorbilidad psiquiátrica es fundamental, y de ello depende la posibilidad de conseguir mejores resultados, así como la reducción de los gastos que se requieren en los tratamientos adecuados para este tipo de pacientes.

\section{OBJETIVO}

En una población con trastornos por uso de drogas (Substance Related Disorder, SRD) señalar la prevalencia de trastornos de personalidad (Personality Disorders, PDs) aplicando el SCID-II.

\section{MATERIALES Y MÉTODOS}

En el Dipartimento per le Dipendenze (Departamento de Adicciones) de Verona (I), siguiendo las directrices de la American Psychiatric Association, se creó y se puso en funcionamiento un protocolo para la valoración de clientes con SRD de tipo tridimensional. La persona que acude al servicio número 1 del Departamento formula una petición de ayuda rellenando el módulo apropiado; a continuación, la persona encargada de la acogida verifica si existe competencia territorial y activa el protocolo de valoración, que consta de una serie de entrevistas con un psicólogo, un asistente social y un médico. El paciente no recibe el resultado de la valoración en cada una de las etapas, sino después de haber superado todos los requisitos.

La tabla $n$. 1 muestra un esquema del protocolo que sigue las directrices marcadas por la American Psychiatric Association para personas que acuden a los servicios para tratamiento de adicciones.

\begin{tabular}{|c|l|l|}
\hline \multicolumn{2}{|c|}{ Tabla 1. Protocolo de valoración } \\
\hline 1 & Petición de ayuda & $\begin{array}{l}\text { La persona formula una petición de ayuda a través del correspondiente módulo } \\
\text { impreso } \\
2\end{array}$ \\
$\begin{array}{l}\text { Criterio de competencia territorial } \\
\text { Aceptación }\end{array}$ & $\begin{array}{l}\text { Protocolo tridimensional } \\
\text { Se propone al cliente seguir el consejo de: una entrevista con asistente social, } \\
\text { una entrevista con psicólogo, una entrevista con médico-psiquiatra, una mues- } \\
\text { tra hematoquímica, un curso de supervivencia. } \\
\text { Reunión con el cliente en la que se le entrega la valoración integrada. }\end{array}$ \\
\hline
\end{tabular}


Esta estrategia permite que el equipo reúna la información y elabore un proyecto terapéutico apropiado para cada caso.

Se trata de un tipo de estrategia muy completa, en la que el psiquiatra elabora su valoración a través de cuatro entrevistas. Durante ellas monitoriza el estatus psíquico del paciente, reúne la anamnesis psiquiátrica y aplica el cuestionario estándar cumplimentado sobre perfiles de personalidad SCID-II. EI SCID II (Structured Clinical Interview for DSM-IV Personality Disorders) es una entrevista semiestructurada que crearon Spitzer y sus colaboradores (1987) para el "screening" de los trastornos de personalidad de Eje $\|^{\circ}$. En la tabla n. 2 se describen las cuatro fases en las que se aplica.

\section{Tabla 2. Protocolo para la aplicación del SCID II}

\begin{tabular}{|c|c|c|}
\hline 1 & Demanda & Explicación de la utilidad de la entrevista y del modo correcto de desarrollarla. \\
\hline 2 & Aceptación & Registro de la aceptación o del rechazo \\
\hline 3 & Factibilidad & $\begin{array}{l}\text { Verificación de existencia de estatus psíquico compatible con la aplicación del SCID, y, en } \\
\text { caso de un estatus psíquico alterado, proyecto de compensación destinado a conseguir un } \\
\text { estatus psíquico compatible. }\end{array}$ \\
\hline 4 & Aplicación & $\begin{array}{l}\text { Se procede a aplicar el cuestionario al cliente explicándole que debe responder a las pre- } \\
\text { guntas intentando ceñirse al sí o al no. Si se equivoca debe señalar con una cruz la res- } \\
\text { puesta equivocada y hacer un círculo en torno a la correcta. } \\
\text { Para responder al las preguntas debe valorar los años pasados y no el presente. }\end{array}$ \\
\hline 5 & Entrega & $\begin{array}{l}\text { Coloquio durante el que se refiere al cliente los eventuales trastornos encontrados y las } \\
\text { eventuales explicaciones del caso. Dado que los PD tienen se caracterizan por manifestar } \\
\text { egosintonía hacia el trastorno, la presencia de algunos trastornos exige que el especialista } \\
\text { conozca y utilice modalidades idóneas de comunicación. }\end{array}$ \\
\hline
\end{tabular}

El protocolo para la valoración psiquiátrica se aplicó a la población de la unidad n. 1 del Departamento de Adicciones de Verona. Se trataba de 115 personas, 88 (77\%) varones, 27 (23\%) mujeres. Todos ellos presentaban características relacionadas con la adicción a los opiáceos. Los individuos se reclutaron entre enero de 1999 y junio de 2002. Para la selección se valoró la existencia de un estatus psíquico compatible con una entrevista. En los casos de clientes con estatus psíquico alterado, bien por consumo de drogas sin prescripción, o por la situación psicopatológica en ese momento, se procedía a compensar la situación, proponiéndole posteriormente de nuevo su inclusión en el proyecto.

El número de individuos a los que se pidió que participasen en la investigación fue de 115; de ellos, aceptaron 114, resultando 113 idóneos a todos los efectos para que se les aplicase el test. Cuando terminó la fase de aplicación, resultaron válidos 112 tests, porque uno de los participantes decidió retirarse del estudio en un segundo momento.

En la tabla y en el gráfico inferiores se reflejan los datos relativos a los individuos a los que se pidió que participasen en la investigación, a la disponibilidad que mostraron, a la factibilidad del cuestionario y a su aplicación.

\section{RESULTADOS}

Las conclusiones de este trabajo tienden a reforzar la hipótesis de una posible relación entre trastornos de personalidad y SUD.
Tabla 3. Criterios seguidos en la aplicación

\begin{tabular}{|lcc|}
\hline Criterios & N. & $\%$ \\
\hline Demanda & 115 & $100 \%$ \\
Disponibilidad & 114 & $99,2 \%$ \\
Factibilidad & 113 & $98,2 \%$ \\
Aplicación & 112 & $97,3 \%$ \\
\hline
\end{tabular}

Fig.1: Criterios seguidos en la aplicación

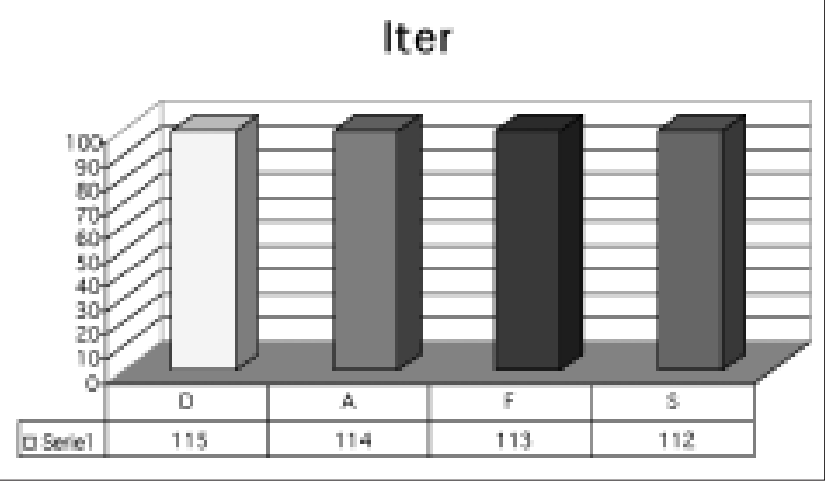

Los trastornos de personalidad (DP) se distribuyeron en tres grupos en base a analogías sintomatológicas. El grupo A incluía a los DP Paranoides, Esquizoides y Esquizotípicos con comportamientos de características comunes de enajenación y excentricidad. El grupo B incluía a los DP Antisociales, Borderline, Histriónicos y Narcisistas, en los que dominan comportamientos emotivos, amplificadores e imprevi- 
sibles. El grupo C incluía a los DP Evitantes, Dependientes y Obsesivo-Compulsivos.

El espectro psicopatológico que reveló nuestra investigación incluía los siguientes trastornos: paranoide, esquizotípico, esquizoide, borderline, antisocial, narcisista, histriónico, evitante, dependiente, obsesivo compulsivo, personalidad negativa y depresivo.

Los trastornos de personalidad más frecuentes fueron: el Borderline (86 individuos sobre 112) con un porcentaje cercano al 76,8\%, seguido del Antisocial 69,6\%, Paranoide 52,7\%, Narcisista 42\%, Obsesivo Compulsivo 40,2\%, Evitante 34,8\%, Esquizoide $34,8 \%$, Dependiente 25,9\%, Esquizotípico 25,9\% y, finalmente, el trastorno que se presentaba con una frecuencia menor era el Histriónico 9,8\%. También se ha evidenciado la presencia de trastornos de Personalidad Negativa $(47,3 \%)$ y de trastornos depresivos $(45,5 \%)$.

En términos generales puede decirse que nuestro estudio confirma el dato de que los PD son uno de los cuadros de comorbilidad psiquiátrica que tienen una presencia mayor en la población de SRD. También se confirma el dato de que el grupo B, y concretamente los trastornos borderline y antisociales son los que presentan mayores porcentajes. Se observa que en nuestra muestra el grupo B comprende tanto los trastornos más frecuentes como el que presenta una frecuencia menor, el histriónico. En lo que respecta al grupo A, el trastorno de paranoia es el de mayor prevalencia, siendo el tercero en términos generales. En relación con la hipótesis de que los aspectos paranoicos estén relacionados con el consumo de drogas sin prescripción, no poseemos datos que permitan esti- maciones definitivas. Sólo podemos señalar que en el momento en que se aplicó el SCID-II el estatus psíquico no mostraba alteraciones, y el cliente estaba en una situación psicopatológica compensada. En el grupo $\mathrm{C}$ los porcentajes son similares a los del grupo A. Los porcentajes de los trastornos negativístas y depresivos son equivalentes, y constituyen el tercer grupo.

Los datos de la tabla n. 4 proceden del análisis de los resultados del SCID II en la población de SRD perteneciente a nuestro servicio.

En el gráfico se representan los datos obtenidos. La imagen ayuda a evidenciar los trastornos de personalidad que mostraron una frecuencia mayor.

\section{Tabla 4. Características principales de la población estudiada.}

\begin{tabular}{lccc|} 
PDs & Grupo & N. & $\%$ \\
\hline Paranoide & A & 59 & $52,7 \%$ \\
Esquizotípico & A & 29 & $25,9 \%$ \\
Esquizoide & A & 39 & $34,8 \%$ \\
Borderline & B & 86 & $76,8 \%$ \\
Antisocial & B & 78 & $69,6 \%$ \\
Narcisista & B & 47 & $42 \%$ \\
Histriónico & B & 11 & $9,8 \%$ \\
Evitante & C & 39 & $34,8 \%$ \\
Dependente & C & 29 & $25,9 \%$ \\
Obsesivo-compulsivo & C & 45 & $40,2 \%$ \\
Personalidad negativa & NO & 53 & $47,3 \%$ \\
Depresivo & NO & 51 & $45,5 \%$ \\
\end{tabular}

Figura 2. aracterísticas principales de la población estudiada.

Personality Disorder

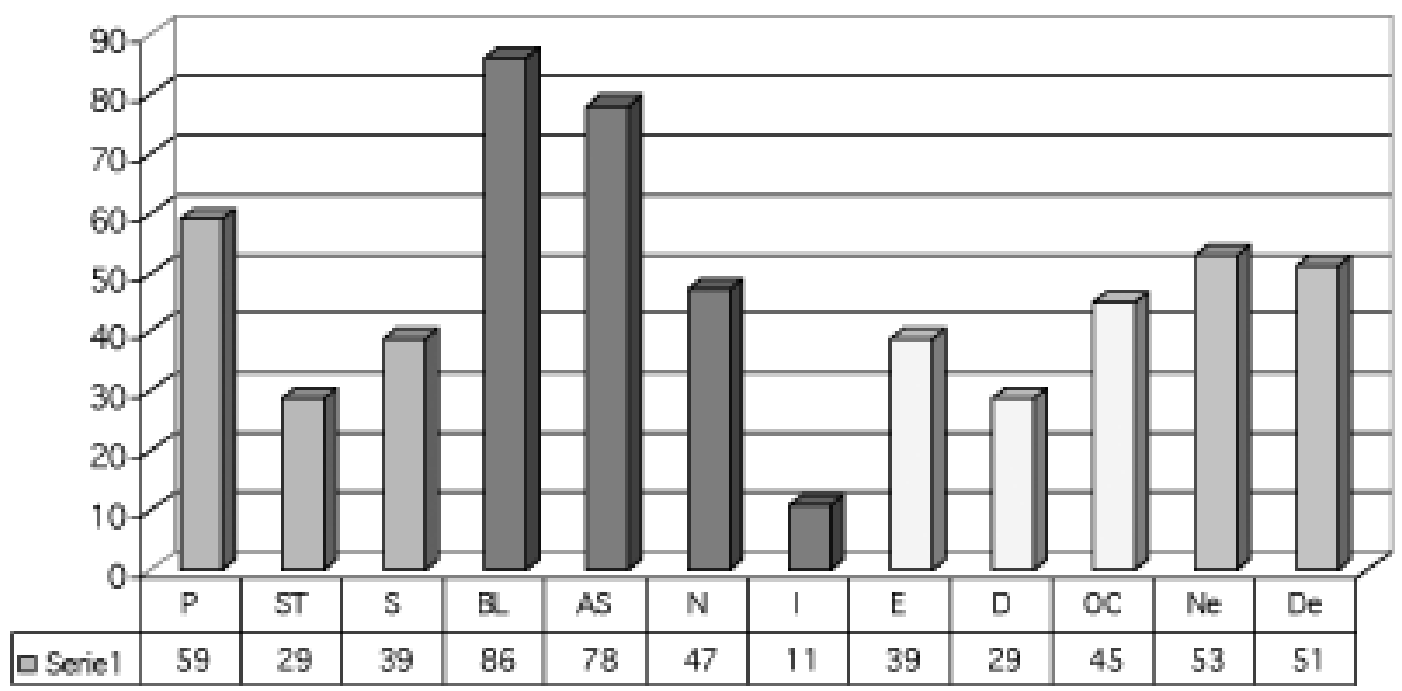




\section{CONCLUSIONES}

Para comprender adecuadamente los resultados del estudio debe recordarse que la categoría PD se introdujo en la gnosografía oficial DSM-ICD a principios de los años 80 . Se trata, por tanto, de una categoría de trastornos que sólo tiene poco más de veinte años de literatura científica. El presente estudio confirma el dato genérico de que entre los 12 PDs, el grupo de mayor prevalencia es el grupo B, y concretamente los trastornos borderline y antisociales.

La posibilidad de comparar la muestra que hemos estudiado con las de otros estudios plantea problemas relacionados con la falta de homogeneidad de dicha muestra. En los estudios italianos, Clerici ha aportado los siguientes datos: en una población adicta a los opiáceos, el 25\% no presentaba doble diagnóstico, el $50 \%$ presentaba un diagnóstico de eje II, y el $25 \%$ restante presentaba un diagnóstico añadido de eje I. En nuestra muestra, sin embargo, el porcentaje bruto de comorbilidad en eje $\|^{\circ}$ supera el $90 \%$, y como no se ha investigado el eje $\mathrm{I}^{\circ}$ no se puede comparar el dato.

El problema de la diferencia de los resultados sobre la prevalencia de los trastornos en eje $1^{\circ}$ y $11^{\circ}$ queda abierto. La causa de esta falta de coincidencia puede atribuirse, en líneas generales, a diversos factores. Por una parte los problemas de homogeneidad de la muestra se relacionan con las propias características de la población SRD; por otra, hay que tener en cuenta que los instrumentos "golden standard" tienen problemas de pesadez, y que su aplicación rutinaria en los servicios territoriales no es fácil, funcionando sólo en casos particulares. Para obtener datos significativos sobre comorbilidad se proponen dos posibles caminos: por una parte, organizar estudios en muchos centros, y por otra seguir la vía contraria de efectuar "screenings" que midan trastornos singulares y específicos. Por ejemplo, investigar sólo el grupo B en vez de todos los PD.

\section{BIBLIOGRAFÍA}

(1) Abram KM, Teplin LA. Co-occurring disorders among mentally ill jail detainees. Implications for public policy. Am Psychol 1991 Oct;46(10):1036-45.

(2) Barrowclough C, Haddock G, Terrier N et al, Cognitivebehavuioral intervention for clients with severe mental illness who have a substance misuse problem. Psychiatric Rehabilitation Skills 4:216-233, 2000.

(3) Caton CLM, The new chronic patient and the system of community care. Hospytal and Community Psychiatry 32 475- 4781981.

(4) Caton CL, Shrout PE, Dominguez B, Eagle PF, Opler LA, Cournos F. Risk factors for homelessness among women with schizophrenia. Am J Public Health 1995 Aug;85(8 Pt 1):1153-6.

(5) Clerici M., et al: Trastornos por uso de drogas y comorbilidad psiquiatrica en un servicio para la toxicodependencia (SERT) durante un periodo de seis años. Adicciones 9, 3, 467-481, 1997. Doppia Diagnosi. Numero monográfico dirigido por M. Clerici; Noos, 20. II Pensiero Scientifico Editore, Roma 2000.

(6) Cuffel BJ, Shumway M, Chouljian TL, MacDonald T. A longitudinal study of substance use and community violence in schizophrenia. J Nerv Ment Dis 1994 Dec;182(12):704-8.

(7) Detrick A, Stiepock V. Treating persons with mental illness, substance abuse, and legal problems: the Rhode Island experience. New Dir Ment Health Serv 1992 Winter;(56):65-77.

(8) Drake RE, Wallach MA, Dual diagnosis: 15 years of progress. Psychiatr Serv. 2000 Sep;51(9):1119-25.

(9) Drake RE, Essock SM, Shaner A, Carey KB, Minkoff K, Kola L, Lynde D, Osher FC, Clark RE, Rickards L. Implementing dual diagnosis services for clients with severe mental illness. Psychiatr Serv 2001 Apr;52(4):469-76.

(10) Ley A, Jeffery DP, McLaren S, Siegfried N. Ley A, Jeffery DP, McLaren S, Siegfried N. Cochrane Database Syst Rev 2000;(4):CD001088.

(11) Haywood TW, Kravitz HM, Grossman LS, Cavanaugh JL Jr, Davis JM, Lewis DA,. Predicting the "revolving door" phenomenon among patients with schizophrenic, schizoaffective, and affective disorders. Am J Psychiatry. 1996 Nov;153(11):1512.

(12) Mead S, Copeland ME. What recovery means to us: consumers' perspectives. Community Ment Health J. 2000 Jun;36(3):329-31.

(13) Meisler N, Blankertz L, Santos AB, McKay C. Impact of assertive community treatment on homeless persons with co-occurring severe psychiatric and substance use disorders. Community Ment Health J 1997 Apr; 33(2): 113-22.

(14) Minkoff K. An integrated treatment model for dual diagnosis of psychosis and addiction. Hosp Community Psychiatry 1989 Oct;40(10):1031-6.

(15) Pepper B, Kirshner MC, Ryglewicz H., The young adult chronic patient: overview of a population. 1981. Psychiatr Serv. 2000 Aug;51(8):996-1000.

(16) Regier DA, Farmer ME, Rae DS, Locke BZ, Keith SJ, Judd LL, Goodwin FK. Comorbidity of mental disorders with alcohol and other drug abuse. Results from the Epidemiologic Catchment Area (ECA) Study. JAMA. 1990 Nov 21;264(19):2511-8.

(17) Ridgely MS, Osher FC, Goldman HH et al, Executive summary: Chronic Mentally III Young Adult With Substance Abuse Problem: A review of research treatment and trainig issue. Baltimore University of Maryland of School of Medicine, Mental Health Research Center.1987.

(18) Rosenberg SD, Goodman LA, Osher FC, Swartz MS, Essock SM, Butterfield MI, Constantine NT, Wolford 
GL, Salyers MP Prevalence of HIV, hepatitis B, and hepatitis $\mathrm{C}$ in people with severe mental illness. Am J Public Health 2001 Jan;91(1):31-7.

(19) Swofford CD, Kasckow JW, Scheller-Gilkey G, Inderbitzin LB. Substance use: a powerful predictor of relapse in schizophrenia. Schizophr Res 1996 May;20(1-2):145-151.
(20) Torrey WC, Wyzik P. The recovery vision as a service improvement guide for community mental health center providers. Community Ment Health J 2000 Apr;36(2):209-16. 